\title{
Photocatalytic activity of mesoporous microbricks of ZnO nanoparticles prepared by the thermal decomposition of bis(2-aminonicotinato) zinc (II)
}

\author{
Keyvan Bijanzad a, Azadeh Tadjarodi a, Omid Akhavan b,c,* \\ a Research Laboratory of Inorganic Materials Synthesis, Department of Chemistry, Iran University of Science and Technology, Narmak, Tehran \\ 16846-13114, Iran \\ b Department of Physics, Sharif University of Technology, P.O. Box 11155-9161, Tehran, Iran \\ c Institute for Nanoscience and Nanotechnology, Sharif University of Technology, P.O. Box 14588-89694, Tehran, Iran
}

\section{A R T I C L E I N F O}

\section{Article history:}

Received 12 December 2014

Accepted 22 January 2015

Published 20 May 2015

\section{Keywords:}

Znic oxide

Bis(2-aminonicotinato) zinc (II)

Mesoporous

Photocatalyst

Nanoparticle

\begin{abstract}
A B S T R A C T
Hollow microblocks of $\left[\mathrm{Zn}(\mathrm{anic})_{2}\right]$, as a novel coordination compound, were synthesized using 2-aminonicotinic acid (Hanic) and zinc (II) nitrate tetrahydrate. The chemical composition of the zinc complex, $\mathrm{ZnC}_{12} \mathrm{H}_{10} \mathrm{~N}_{4} \mathrm{O}_{4}$, was determined by Fourier transform infrared (FTIR) spectroscopy and elemental analysis. The synthesized zinc complex was used as a precursor to produce $\mathrm{ZnO}$ nanostructures by calcination at $550{ }^{\circ} \mathrm{C}$ for $4 \mathrm{~h}$. Morphological studies by scanning electron microscopy and transmission electron microscopy revealed the formation of porous microbricks of $\mathrm{ZnO}$ nanoparticles. $\mathrm{N}_{2}$ adsorption-desorption analysis showed that the obtained $\mathrm{ZnO}$ microbricks possess a mesoporous structure with a surface area of $8.13 \mathrm{~m}^{2} / \mathrm{g}$ and a pore size of $22.6 \mathrm{~nm}$. The X-ray diffraction pattern of the final product proved the formation of a pure $\mathrm{ZnO}$ composition with a hexagonal structure. Moreover, FTIR analyses showed that the 2-aminonicotinic acid ligand peaks were absent after the calcination step. Diffuse reflectance spectroscopy was used to determine the band gap energy of the produced $\mathrm{ZnO}$ and it was about $3.19 \mathrm{eV}$. To investigate the photocatalytic activity of the porous $\mathrm{ZnO}$ nanostructure, a series of photocatalytic tests were carried out to remove Congo red, as a representative toxic azo dye, from aqueous solution. The results show that the product can be used as an efficient photocatalyst for waste water treatment with high degradation efficiency.
\end{abstract}

(C) 2015, Dalian Institute of Chemical Physics, Chinese Academy of Sciences.

Published by Elsevier B.V. All rights reserved.

\section{Introduction}

Recently, considerable attention has been given to the synthesis of diverse zinc oxide nanostructures because of their broad range of applications in science and technology such as in rubbers [1], antibacterial nanomaterials [2,3], LEDs [4], transparent transistors [5], solar cells [6], photocatalytic reductants [7], memory devices [8], graphene semiconductors [9], organic reactions catalysts $[10,11]$ and photocatalysts [12-14]. ZnO is a wide band gap (ca. $3.37 \mathrm{eV}$ ) n-type semicon- ductor with a hexagonal wurtzite structure. The production of porous $\mathrm{ZnO}$ with a high specific surface area will add to its many advantages. In the mesoporous structure of a semiconductor, the channels can act as a light-transfer path for the introduction of incident photons onto the inner surface of the photocatalyst and this enhances its activity [15]. Additionally, the degradation efficiency of a porous photocatalyst might be enhanced by its adsorption ability because of an increase in the probability of a surface reaction with pollutant molecules [16]. Additionally, the morphology of nanomaterials also has a great

\footnotetext{
*Corresponding author. Tel/Fax:+98-21-66022711; E-mail: oakhavan@sharif.edu 
impact on their properties and hence their applications [17]. Contrary to expectations, a large shrinkage of the porous materials obtained from the template-directed method occurs during heat treatment. This phenomenon causes the collapse of mesoporous structures [18]. Therefore, new methods are required for the preservation of the mesoporous framework of materials and for a repeatable route to fabricate mesoporous structures.

A suitable method for the fabrication of metal oxide nanostructures is the thermal decomposition of metal complexes. To date, different architectures of 2-aminonicotinic acid (Hanic) coordination compounds have been synthesized as a consequence of its specific topology. $[\mathrm{Tb}$ (anic) 3$] \cdot 0.5 \mathrm{Hanic} \cdot 7 \mathrm{H}_{2} \mathrm{O}$ with photoluminescence properties [19], $\left[\mathrm{Ag}_{2}-\mu-\mathrm{O}, \mathrm{O}^{\prime}(\mathrm{anic})_{2}\left(\mathrm{NO}_{3}\right)_{2}\right]_{n}$ with high antibacterial activity [20] and $\left[\mathrm{TlMe}_{2}(\mathrm{anic})\right]_{n}$ [21] are examples of coordination compounds that contain 2-aminonicotinic acid. To the best of our knowledge, coordination compounds between zinc and 2-aminonicotinic acid have not been reported.

It has been reported that $\mathrm{ZnO}$ nanoparticles could be generated from solvothermally prepared zinc (II) coordination polymers containing aromatic dicarboxylic acids [22]. A zinc terephthalate metal-organic framework has been reported to give porous cubic-shaped $\mathrm{ZnO}$ particles via pyrolytic conversion [23]. The mechanochemical process, as a green solid state synthetic method, was used to prepare $\mathrm{Zn}\left(\mathrm{C}_{2} \mathrm{O}_{4}\right) \cdot 2 \mathrm{H}_{2} \mathrm{O}$, which was then calcined to give $\mathrm{ZnO}$ porous nanostructures [24]. Zinc hydroxide carbonate was obtained with the assistance of glycine and polyvinyl pyrrolidone to produce a nest-like 3D ZnO nanoporous material using post heat treatment [25]. Furthermore, ZnO nanostructures were synthesized by pulsed laser deposition [26] and radio frequency magnetron sputtering [27].

Congo red (CR) is one of the most popular secondary diazo dyes and it may cause cancer in humans because of its toxic metabolite benzidine. Its highly colored effluents have low biological oxygen demand and high chemical oxygen demand and they also contain high quantities of dissolved solids [28]. In this research, [Zn(anic) 2] was synthesized by chemical precipitation and used as a precursor to prepare $\mathrm{ZnO}$ nanoparticles after heat treatment. We investigated its photocatalytic activity toward the photodegradation of CR.

\section{Experimental}

\subsection{Chemicals and synthesis}

All the reagents were purchased from the Merck Company in Germany and used without further purification. Congo red (sodium 3,3'-([1,1'-biphenyl]-4,4'-diyl)bis(4-aminonaphthalene-1-sulfonate) was used as a model azo dye. Deionized wa- ter was used to prepare all the solutions. For the synthesis, a 3 mmol ethanolic solution of 2-aminonicotinic acid $(30 \mathrm{~mL})$ and 3 mmol $\mathrm{KOH}$ aqueous solution were mixed until the $\mathrm{pH}$ of the solution was 7, as measured by a Metrohm pH meter. $1.5 \mathrm{mmol}$ $\mathrm{Zn}\left(\mathrm{NO}_{3}\right)_{2} \cdot 4 \mathrm{H}_{2} \mathrm{O}$ aqueous solution $(20 \mathrm{~mL})$ was then added dropwise to this mixture while stirring for $20 \mathrm{~min}$. The resultant white precipitate of the zinc coordination compound (with a decomposition temperature $>350^{\circ} \mathrm{C}$ ) was filtered, washed and dried at room temperature overnight. Ultimately, it was calcined at $550{ }^{\circ} \mathrm{C}$ for $4 \mathrm{~h}$ to give the $\mathrm{ZnO}$ nanostructures. A schematic representation of the reaction is shown in Scheme 1. Commercially available particulate $\mathrm{ZnO}$ (particle size: $200 \mathrm{~nm}$ ) and $\mathrm{TiO}_{2}$ powder (anatase, particle size: $30 \mathrm{~nm}$ ) were also investigated to compare their photocatalytic activity with that of the synthesized $\mathrm{ZnO}$ nanoparticles.

\subsection{Characterization}

To characterize the samples the X-ray diffraction (XRD) pattern of the $\mathrm{ZnO}$ product was obtained using a JEOL diffractometer with monochromatic $\mathrm{Cu} K_{\alpha}$ radiation $(\lambda=0.15418 \mathrm{~nm})$. Diffuse-reflectance spectra (DRS) were obtained using a Shimadzu (MPC-2200) spectrophotometer. Fourier transform infrared spectra (FTIR) were obtained using a Shimadzu-8400S spectrometer in the range of $400-4000 \mathrm{~cm}^{-1}$ using $\mathrm{KBr}$ pellets. Scanning electron microscopy (SEM) was performed with a VEGA/TESCAN microscope at an accelerating voltage of 30.00 $\mathrm{kV}$. Field emission scanning electron microscopy (FESEM) was performed using a $\Sigma$ IGMA series microscope at an accelerating voltage of $15 \mathrm{kV}$, and this was purchased from the Zeiss Company, Germany. Transmission electron microscopy (TEM) was carried out using an EM10C-100 kV series microscope that was purchased from the Zeiss Company, Germany. The surface area of the product was obtained using the Brunauer-Emmett-Teller (BET) technique with Micromeritics (Gemini) in the range of relative pressures from $0.0-1.0$. Before the measurement, the sample was degassed at $200{ }^{\circ} \mathrm{C}$ for $2 \mathrm{~h}$. Additionally, the pore size distribution was determined from the desorption branch of the isotherm curve using the Barrett-Joyner-Halenda (BJH) model. Elemental analysis was performed with a CHN Rapid Heraeus elemental analyzer. The UV-Vis absorption studies were performed at room temperature in the wavelength range of 190-800 $\mathrm{nm}$ on a UV-Vis spectrophotometer (Shimadzu UV-1700). The $\mathrm{pH}$ was measured using a Metrohm $\mathrm{pH}$ meter type 1.712.0010 that was purchased from Metrohm Herisau, Switzerland.

\subsection{Photocatalytic test}

The azo dye CR was used as the model pollutant to evaluate the photocatalytic activity of the prepared $\mathrm{ZnO}$. The photodeg-

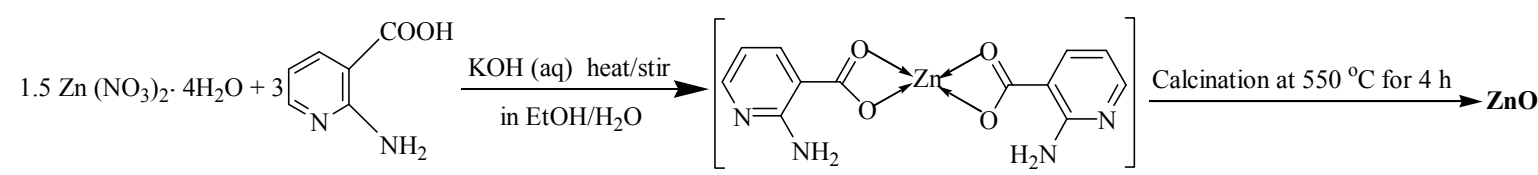

Scheme 1. Formation reaction for the zinc complex and for zinc oxide. 
radation experiments were carried out under the following conditions: $0.02 \mathrm{~g}$ of the $\mathrm{ZnO}$ nanostructure as the photocatalyst was introduced to a $50 \mathrm{~mL}$ dye aqueous solution with an initial concentration of $50 \mathrm{mg} / \mathrm{L}$ at room temperature and at neutral $\mathrm{pH}$. The resultant suspension was stirred using a magnetic stirrer for $1 \mathrm{~h}$ in the dark to establish an adsorption-desorption equilibrium. The concentration of the residual dye was measured using a UV-Vis spectrophotometer at the appropriate wavelength that corresponds to the maximum absorption of CR (498 nm). The reaction vessel was then subjected to light irradiation in the photo-reactor to carry out the photodegradation process. Light irradiation was supplied by a $500 \mathrm{~W}$ high-pressure mercury-vapor lamp $(\lambda=546.8 \mathrm{~nm})$ that was mounted $10 \mathrm{~cm}$ from the reaction solution. The mercury lamp (HWL $500 \mathrm{~W} 225 \mathrm{~V}$ E40) was purchased from the OSRAM Company in China and the short wavelength components of the supplied light $(\lambda<350 \mathrm{~nm})$ were cut off using a filter. At given irradiation time intervals portions of the suspension were taken from the reaction vessel, centrifuged at $2000 \mathrm{rpm}$ for $5 \mathrm{~min}$ and analyzed by UV-Vis spectrophotometer.

\section{Results and discussion}

\subsection{Compositional characterization}

Elemental analysis calculations verified that a zinc coordination compound with the empirical formula $\mathrm{ZnC}_{12} \mathrm{H}_{10} \mathrm{~N}_{4} \mathrm{O}_{4}$ was synthesized. The carbon, hydrogen and nitrogen mass percentages were: C $42.43 \%$, $\mathrm{H} 2.86 \%$ and $\mathrm{N} 16.39 \%$, and these are similar to the calculated values (C $42.43 \%, \mathrm{H} 2.95 \%$ and $\mathrm{N}$ $16.86 \%)$.

Fourier transform infrared (FTIR) spectroscopy was used to characterize the products. The FTIR spectra of the zinc complex and the zinc oxide are shown in Fig. 1. All the peaks related to the 2-aminonicotinic acid functional groups were present in the spectrum of the zinc complex (Fig. 1(1). Aromatic C-H bending vibrations were observed at $750-835 \mathrm{~cm}^{-1}$ and the bands from the $\mathrm{C}-\mathrm{N}$ and $\mathrm{C}-\mathrm{O}$ vibrations were clearly present at $\sim 1257$ $\mathrm{cm}^{-1}$. The peaks at 1404 and $1541 \mathrm{~cm}^{-1}$ are assigned to the $\mathrm{C}=\mathrm{C}$ stretching and $\mathrm{N}-\mathrm{H}$ bending vibrations, respectively. In the zinc complex, the asymmetric vibrational mode of the carboxylate

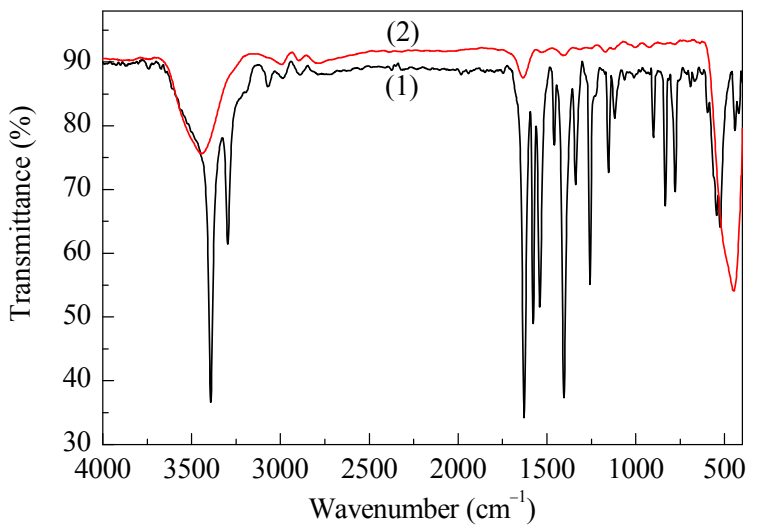

Fig. 1. FTIR spectra of (1) the zinc complex and (2) the $\mathrm{ZnO}$ nanostructure. group shifted to lower wavenumber (at $1627 \mathrm{~cm}^{-1}$ ) and the symmetric vibrational mode of the carboxylate group shifted to higher wavenumber (at $1562 \mathrm{~cm}^{-1}$ ) compared to the free ligand at 1704 and $1548 \mathrm{~cm}^{-1}$, respectively. This reveals coordination through the carboxylate group. The peaks at 3296 and 3392 $\mathrm{cm}^{-1}$ are representative of hydrogens in the amine group. No shift to lower wavenumbers was observed for these peaks after coordination and this implies that 2-aminonicotinic acid was not coordinated to the metal through the nitrogen of the amine group. Because of the distance of the pyridyl nitrogen, it was less likely to be coordinated to the metal $[19,29]$. Based on the obtained results, the ligand acts as a chelate and coordinates to zinc via two oxygen atoms of the carboxylate groups, which might in turn participate in hydrogen bonding. After calcination, all the characteristic peaks of the ligand disappeared and only a peak at $428 \mathrm{~cm}^{-1}$, which is attributed to the $\mathrm{Zn}-0$ bond remained (see Fig. 1(2)).

The XRD technique was used to prove the formation of the $\mathrm{ZnO}$ phase after the calcination process. As shown in Fig. 2, all the reflections were well indexed to a hexagonal wurtzite phase of a $\mathrm{ZnO}$ pattern with a space group of $\mathrm{P} 63 \mathrm{mc}$ and lattice parameters of $a=0.325 \mathrm{~nm}, b=0.325 \mathrm{~nm}$ and $c=0.521 \mathrm{~nm}, \alpha=$ $90^{\circ}, \beta=90^{\circ}$ and $\gamma=120^{\circ}$ (JCPDS card no. 00-036-1451). The diffraction peaks at $2 \theta$ values of $31.77^{\circ}, 34.42^{\circ}, 36.25^{\circ}, 47.53^{\circ}$, $56.60^{\circ}, 62.86^{\circ}, 67.96^{\circ}$ and $69.10^{\circ}$ match the respective (100), (002), (101), (102), (110), (103), (112) and (201) planes of hexagonal $\mathrm{ZnO}$ well. The exact number of peaks indicates that the final product is of high purity and that it is a single phase.

\subsection{Morphological and optical studies}

The morphology of the products was studied by SEM and TEM, as shown in Fig. 3. The structure of the zinc complex consisted of hollow microblocks (Fig. 3(a) and (c)). The calcination process resulted in porous microbricks that comprise $\mathrm{ZnO}$ nanoparticles (Fig. 3(d) and (f)). ZnO nanoparticles formed the body of the porous $\mathrm{ZnO}$ structure. It can be concluded that the pores were formed as a consequence of the liberation of volatile gases such as $\mathrm{CO}_{2}, \mathrm{H}_{2} \mathrm{O}$ and $\mathrm{NO}_{x}$ during the thermal decomposition of the complex. Gas release gave rise to the formation of $\mathrm{ZnO}$ nanoparticles in the walls of the microbricks, which finally provided a peculiar mesoporous structure. The TEM

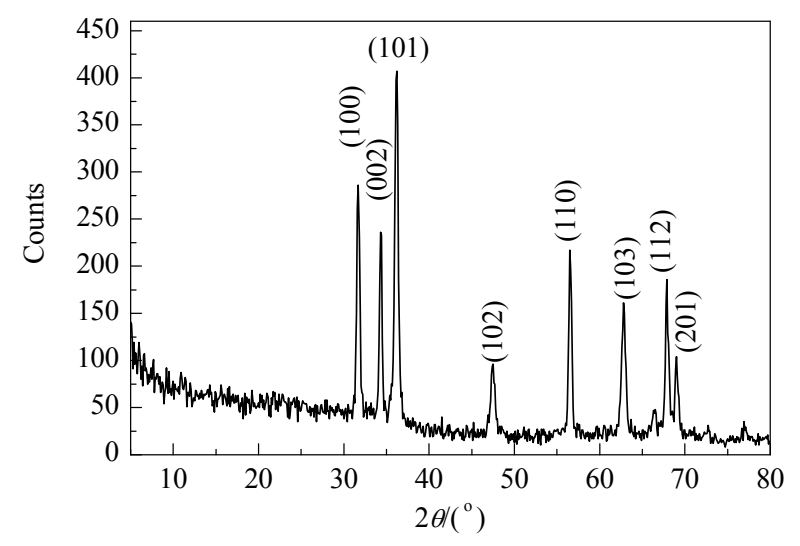

Fig. 2. XRD pattern of the $\mathrm{ZnO}$ nanostructure. 

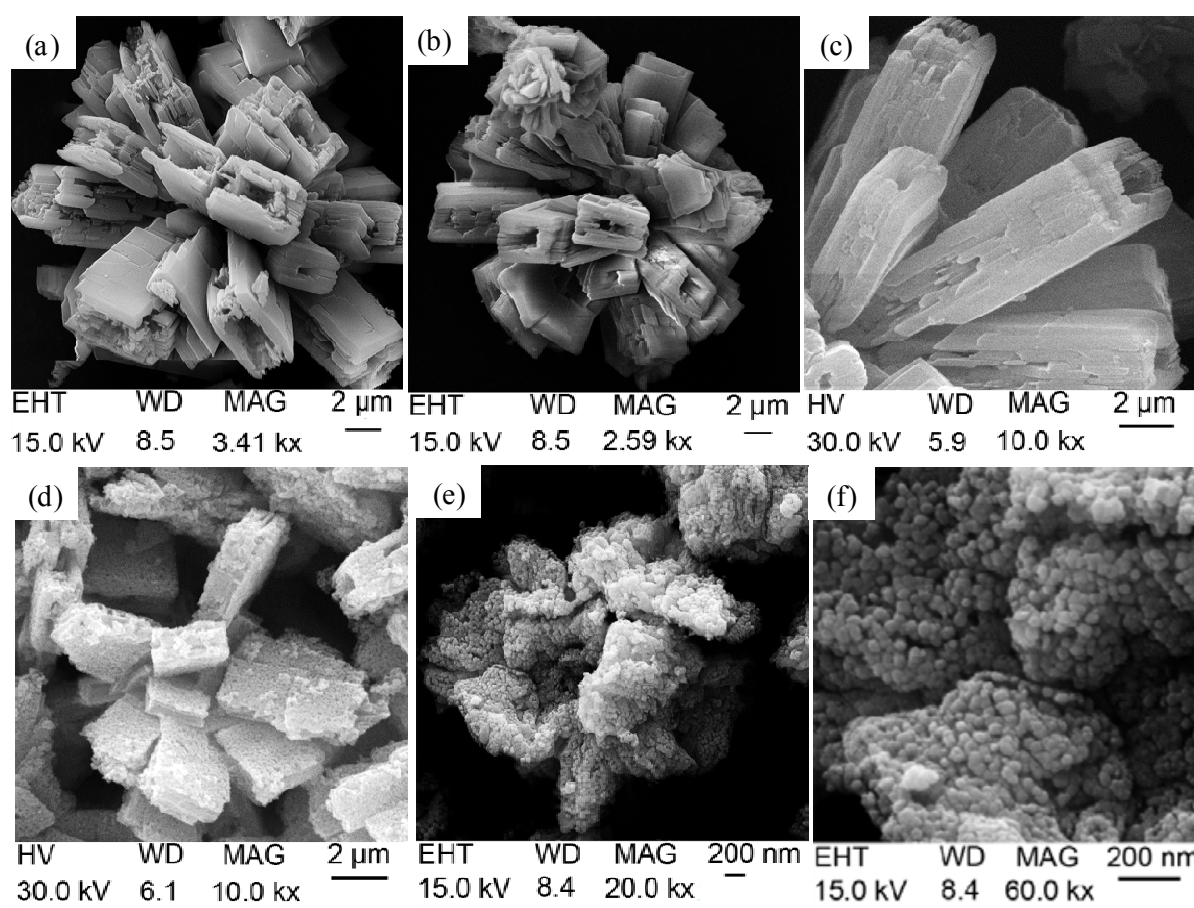

$30.0 \mathrm{kV} \quad 5.9 \quad 10.0 \mathrm{kx}$
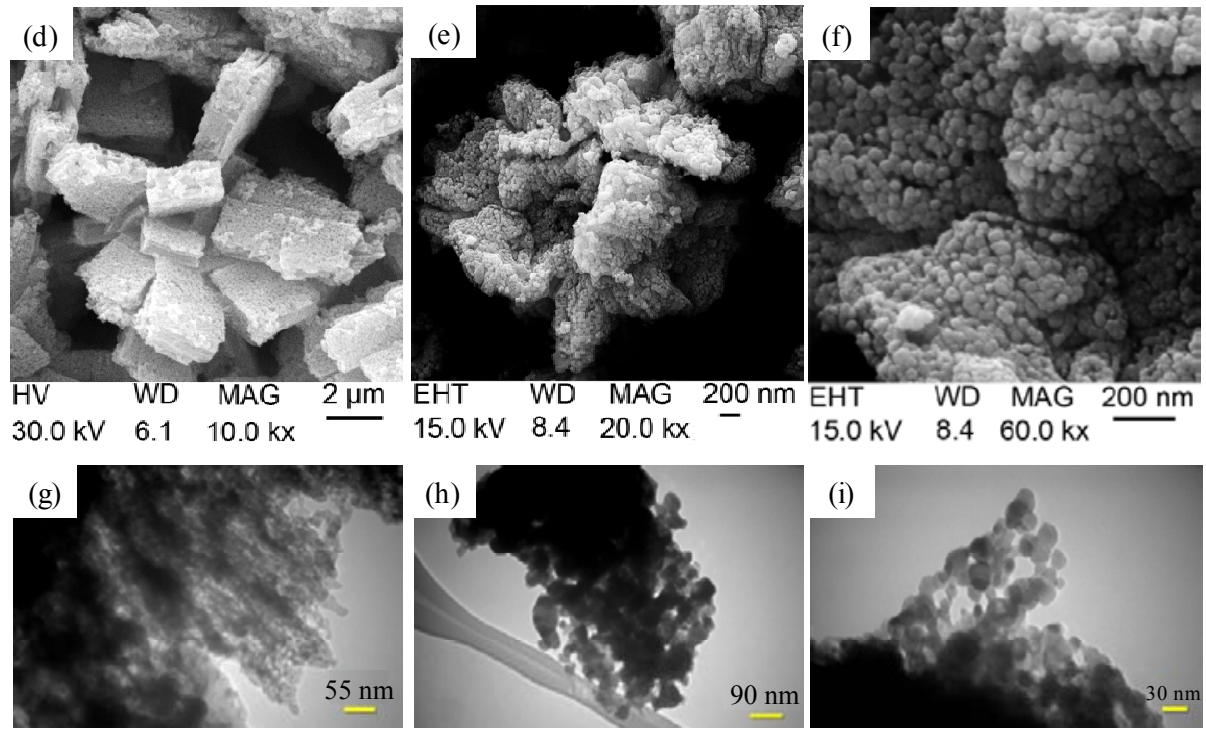

Fig. 3. SEM images of hollow microblocks of the $\mathrm{ZnC}_{12} \mathrm{H}_{10} \mathrm{~N}_{4} \mathrm{O}_{4}$ complex in ((a) and (b)) a wide and ((c)) a close up window and ((d) and (e)) $\mathrm{ZnO}$ microbricks containing ((f)) ZnO nanoparticles; TEM images ((g)-(i)) of the mesoporous structure of the ZnO nanoparticles.

images in Fig. 3(g) and (i) revealed that the mesoporous structure of $\mathrm{ZnO}$ consisted of nanoparticles, which is in agreement with the SEM images. It is clear that the porosity mainly comes from the interparticle porosity. The transparent regions of the TEM images are indicative of pores in which dye molecules are easily trapped. From Fig. 3(i), ZnO nanoparticles with a size of $\sim 30 \mathrm{~nm}$ are clearly observed. The production-calcination experiments were repeated and the same morphology was obtained. Therefore, this is a suitable and repeatable method for the preparation of mesoporous microbricks of $\mathrm{ZnO}$ nanoparticles.

Optical absorption data near the band edge were used to calculate the band gap of the product using the following equation:

$$
\alpha h v=A\left(h v-E_{\mathrm{g}}\right)^{n / 2}
$$

where $\alpha, v, A$, and $E_{\mathrm{g}}$ are the absorption coefficient, light frequency, proportionality constant, and band gap energy, respectively. Here, $n$ identifies whether the transition in a semiconductor is direct $(n=1)$ or indirect $(n=4)$. The values of $n$ and $E_{\mathrm{g}}$ can be estimated as follows: (1) assume an approximate $E_{\mathrm{g}}$ value, $\ln (\alpha h v)$ versus $\ln \left(h v E_{\mathrm{g}}\right)$ should be plotted and the value of $n$ can be obtained from the slope of the straightest line near the band edge, (2) $(a h v)^{2 / n}$ versus $h v$ should be plotted, and the band gap $E_{\mathrm{g}}$ can be calculated by extrapolating the straightest line to the $h v$ axis intercept [30]. The value of $n$ for $\mathrm{ZnO}$ was estimated to be 1 and, therefore, it was a direct gap semiconductor. The band gap energy of the $\mathrm{ZnO}$ nanoparticles was calculated to be about $3.19 \mathrm{eV}$ (Fig. 4) as obtained from the UV-Vis diffuse reflectance spectrum (see inset of Fig. 4).

Information about the specific surface area and the pore size distribution of the $\mathrm{ZnO}$ nanostructure was obtained by $\mathrm{N}_{2}$ adsorption-desorption analysis (BET). The adsorption and desorption isotherm curves are shown in Fig. 5. The BJH model obtained using the desorption branch of the nitrogen isotherm

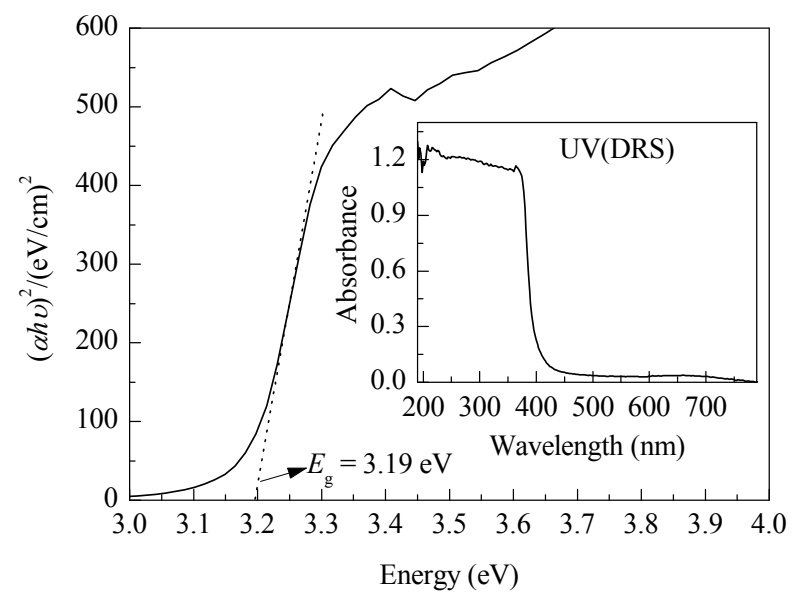

Fig. 4. Plot of $(\alpha h v)^{2} v s$. $h v$ for $\mathrm{ZnO}$ (inset shows the UV-Vis DRS). 


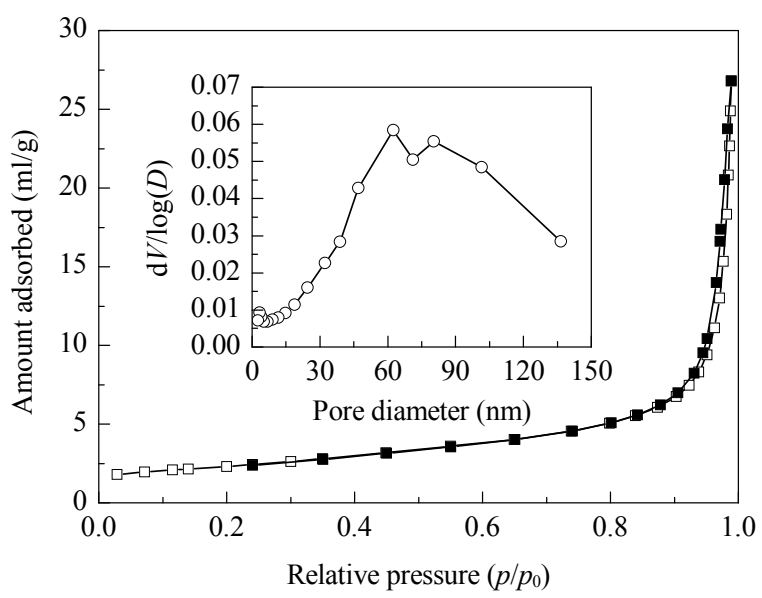

Fig. 5. Nitrogen adsorption $(\bullet)$-desorption $(\boldsymbol{\Delta})$ isotherms for the microbricks of the $\mathrm{ZnO}$ nanoparticles (inset shows a BJH plot of this product).

was also used to evaluate the pore size distribution (inset of Fig. 5). Studies have revealed a distinct hysteresis loop in the range of $0<p / p_{0}<1$ corresponding to diagram $\mathrm{V}$ with a $\mathrm{H} 3$ type loop suggesting the presence of mesopores in the microbricks of the $\mathrm{ZnO}$ nanoparticles. The BET surface area was calculated to be $8.13 \mathrm{~m}^{2} / \mathrm{g}$. The calculated pore size distribution shows that the size of the mesopores are not uniform but are centered at $22.6 \mathrm{~nm}$.

\subsection{Photocatalytic activity}

\subsubsection{Photocatalytic experiment}

The photocatalytic performance of the $\mathrm{ZnO}$ nanoparticles was investigated by the degradation of the toxic azo dye CR from aqueous solution. The removal efficiency was calculated using the following equation:

$$
\text { Removal efficiency }(\%)=\frac{C_{0}-C_{t}}{C_{0}} \times 100
$$

where $C_{0}$ is the initial dye concentration, $\mathrm{C}_{\mathrm{t}}$ is the dye concentration after treatment at different time intervals. The control tests revealed that the maximum CR removal efficiency after 3 $\mathrm{h}$ of illumination was $96 \%$. A slight degradation of the dye pol-

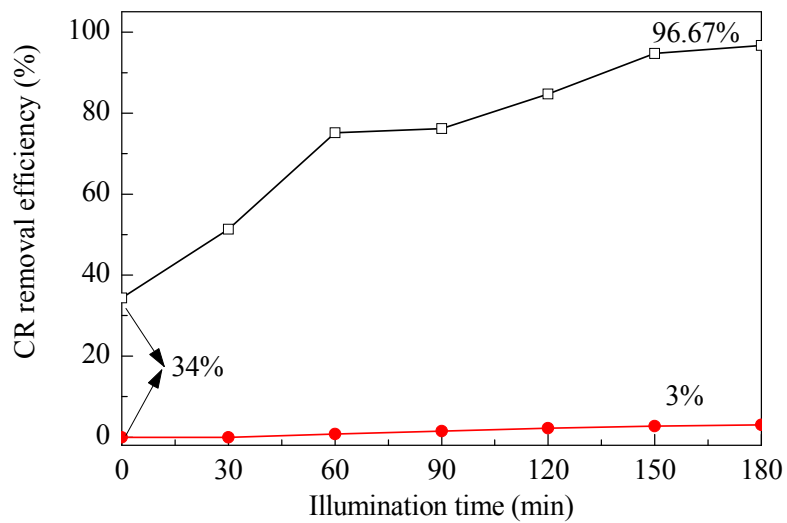

Fig. 6. Decolorizing efficiency toward the CR dye solution by the $\mathrm{ZnO}$ nanostructure.

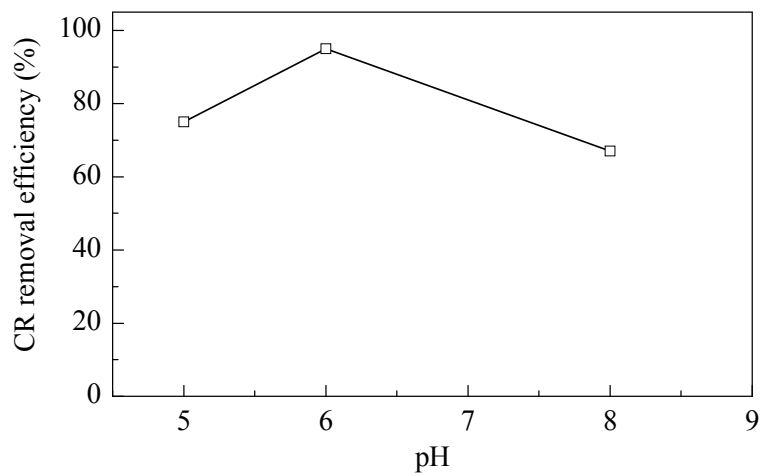

Fig. 7. Effect of $\mathrm{pH}$ on the removal efficiency.

lutant was observed in the absence of photocatalyst under light irradiation (3\%). Adsorption-desorption in the dark showed a CR concentration decrease of $34 \%$. This might come from the porous structure of the product and hence an increase in available adsorption sites on the surface of the catalyst (Fig. 6). By placing the reaction vessel under light irradiation, a superior removal efficiency of dye molecules was observed, which obviously comes from the photocatalytic activity of the $\mathrm{ZnO}$ nanostructure. We found degradation values close to $100 \%$ after $3 \mathrm{~h}$ of illumination, which confirms the superior performance of the synthesized $\mathrm{ZnO}$ nanostructure toward the photodegradation of CR under light illumination in a short time. The effect of solution $\mathrm{pH}$ on the degradation efficiency of the pollutant by the $\mathrm{ZnO}$ nanostructure was also evaluated by adjusting the $\mathrm{pH}$ of the aqueous solution to 5-8 using $\mathrm{HCl}$ or $\mathrm{NaOH}$ solutions $(0.01 \mathrm{~mol} / \mathrm{L})$. The results showed that the highest $\mathrm{CR}$ removal efficiency occurs at a $\mathrm{pH}$ of 6 , which is close to the neutral $\mathrm{pH}$ (Fig. 7). Therefore, the neutral $\mathrm{pH}$ was selected as an appropriate $\mathrm{pH}$ for the photocatalytic tests. The effect of the amount of photocatalyst on the removal of the CR dye is shown in Fig. 8. Different quantities of the prepared $\mathrm{ZnO}$ nanostructure (0.005-0.025 g) were added to a solution containing $50 \mathrm{~mL}$ of CR dye at a concentration of $50 \mathrm{mg} / \mathrm{L}$ at neutral $\mathrm{pH}$. The maximum efficiency was obtained when $0.02 \mathrm{~g}$ of the $\mathrm{ZnO}$ nanoparticles was used. Consequently, this amount of catalyst was chosen as the optimum amount for further investigations into degradation activity.

The photocatalytic activity of the as-prepared $\mathrm{ZnO}$

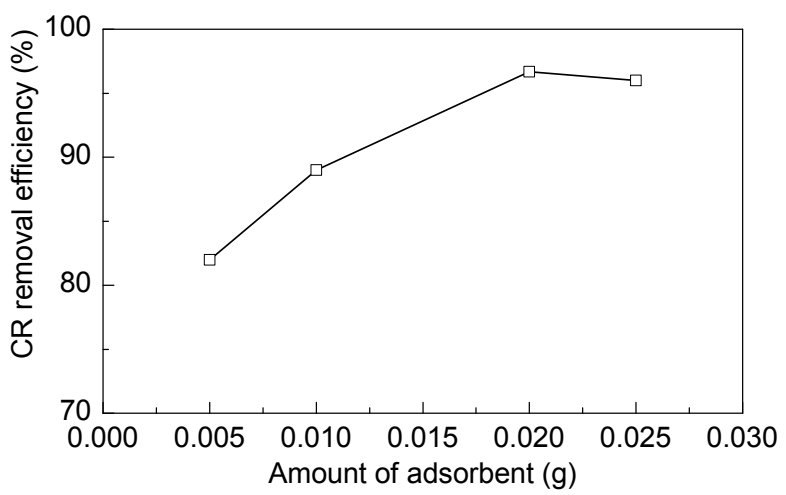

Fig. 8. Effect of catalyst amount on the degradation of CR in aqueous solution. 


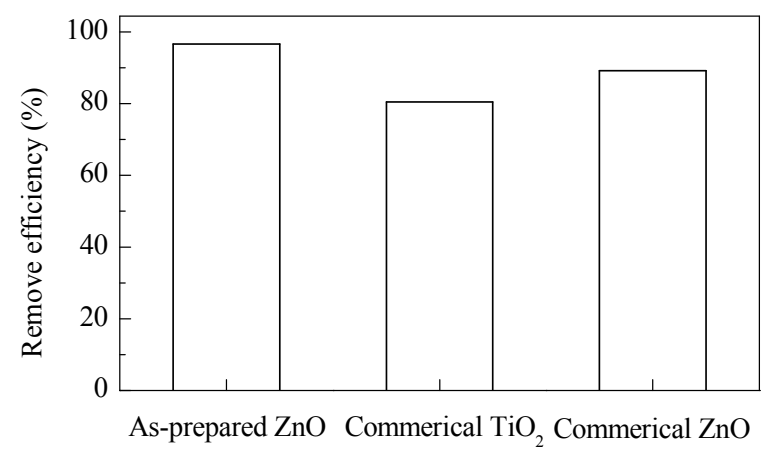

Fig. 9. Photocatalytic activities of the as-prepared $\mathrm{ZnO}$ nanostructure and commercial $\mathrm{TiO}_{2}$ and $\mathrm{ZnO}$ powders.

nanostructure was compared with commercial $\mathrm{TiO}_{2}$ and $\mathrm{ZnO}$ photocatalyst powders and their removal efficiencies were 96.67\%, 80.51\% and 89.15\%, respectively. As shown in Fig. 9, the as-prepared $\mathrm{ZnO}$ nanostructure had the highest activity under the same conditions.

\subsubsection{Photocatalytic reaction kinetics}

To evaluate the catalytic kinetics, the decolorization rate of the CR dye was recorded at different time. The results indicate a linear relationship between $\operatorname{Ln}\left(C_{0} / C_{\mathrm{t}}\right)$ and the reaction time $t$ (Fig. 10), which can be described by a first-order kinetic model (Eq. 3).

$$
\operatorname{Ln}\left(\frac{C_{0}}{C_{t}}\right)=k t
$$

where $C_{0}$ is the initial concentration ( $\left.\mathrm{mmol} / \mathrm{L}\right), C_{t}$ is the instantaneous concentration of the dye at different intervals of illumination and the slope, $k$, is the apparent rate constant. From the graph, the rate constant of the photodegradation reactions for the CR dye is $0.0163 \mathrm{~min}^{-1}$.

\subsubsection{Photodegradation mechanism}

The photocatalytic activity of semiconductors is driven by hydroxyl $(\cdot \mathrm{OH})$ and superoxide $\left(\mathrm{O}_{2}{ }^{\bullet-}\right)$ radicals because of the formation of electron/hole pairs as a result of light irradiation. As the catalyst surface is photoexcited, electrons $\left(\mathrm{e}^{-}\right)$and holes $\left(\mathrm{h}^{+}\right)$are produced in the conduction and valance bands of the photocatalyst, respectively. The mesoporous channels pro-

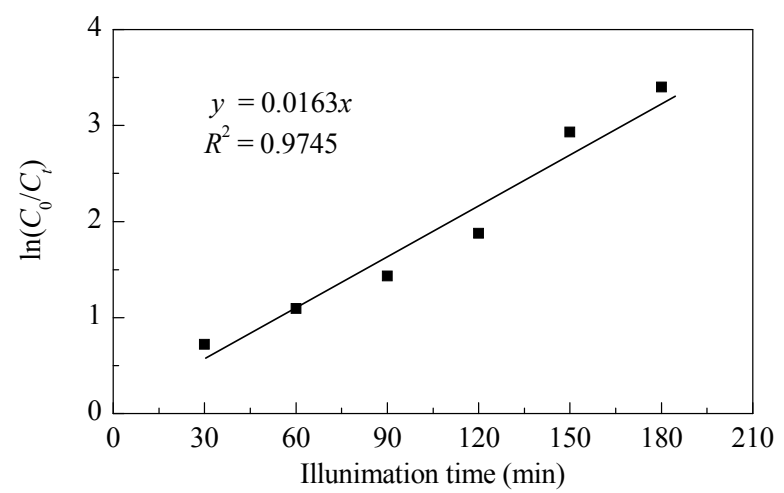

Fig. 10. Photocatalytic kinetics for the degradation of CR molecules upon light illumination. duced from the interparticle connection of $\mathrm{ZnO}$ nanoparticles improve adsorption ability and provide efficient transport pathways for reactant molecules and products [31,32]. The CR molecules can thus be directly oxidized by holes and radicals inside these pores [33]. However, with its mesoporous structure, the pure crystalline phase of $\mathrm{ZnO}$ may have a great impact on its photocatalytic performance [34].

On the other hand, surface hydroxyl groups or chemisorbed water molecules on $\mathrm{ZnO}$ nanostructures remarkably influence the activity by two distinct routes: scavenging the holes and as adsorption sites for reactants and intermediates [35]. According to the literature [36], the oxygen molecules dissolved in the solution capture electrons in the conduction band and the holes in the valence band are captured by surface hydroxyl groups on the catalysts to generate hydroxyl radicals. These subsequently oxidize the adsorbed dye molecules. Additionally, as the formation rate of the $\cdot \mathrm{OH}$ radicals increase a higher separation efficiency is achieved for the electron-hole pairs. Furthermore, it has been shown that surface hydroxyl groups significantly affect photocatalytic activity because these groups can be trapped by the holes generated by UV light irradiation to produce hydroxyl radicals [37]. As a result of the special morphology of the semiconductor numerous available contact sites are available. These adsorbed dye molecules on the active sites of the semiconductor surface harvest electrons in the conduction band of the photocatalyst leading to the formation of radical anions from the dye molecules and the further oxidation and degradation of CR molecules.

To detect active species during the photocatalytic reaction, hydroxyl radicals $\left(\bullet^{\circ} \mathrm{OH}\right)$, superoxide radicals $\left(\mathrm{O}_{2}{ }^{-}\right)$and holes $\left(\mathrm{h}^{+}\right)$were analyzed by adding isopropanol (IPA; $\left.1 \mathrm{mmol} / \mathrm{L}\right)(\mathrm{a}$ quencher of ${ }^{\circ} \mathrm{OH}$ ), $p$-benzoquinone (BQ) (a quencher of $\mathrm{O}_{2^{-}}$) and disodium ethylenediaminetetraacetate (EDTA) (a quencher of $\mathrm{h}^{+}$). This method is identical to the reported photocatalytic activity test [38]. Fig. 11 shows the results of the active species trapping experiment during the photocatalytic reaction. The photocatalytic degradation of CR decreased with the addition of all the quenching agents. Therefore, it can be concluded that $\mathrm{h}^{+}$, $\mathrm{O}_{2} \cdot-$ and $\cdot \mathrm{OH}$ contribute as active species of $\mathrm{ZnO}$ in aqueous solution under light irradiation. Nevertheless, the CR decomposition rate decreased significantly upon the addition of EDTA (1 mmol/L), indicating that holes play a critical role in the CR photodegradation process.

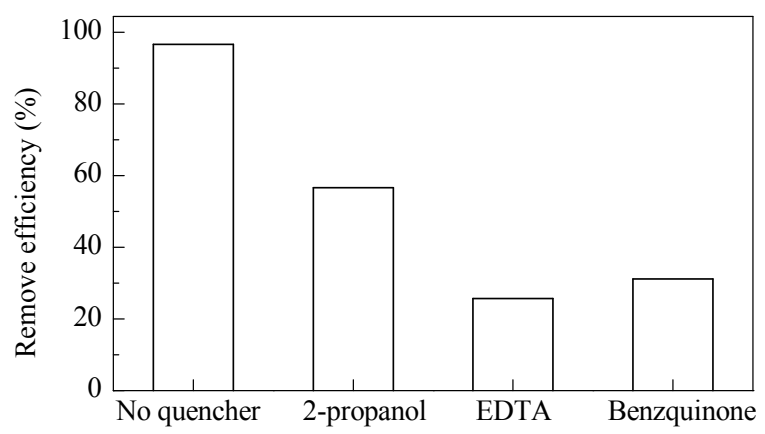

Fig. 11. Photocatalytic degradation of $\mathrm{CR}$ over the $\mathrm{ZnO}$ photocatalyst alone and with the addition of IPA, EDTA or BQ. 


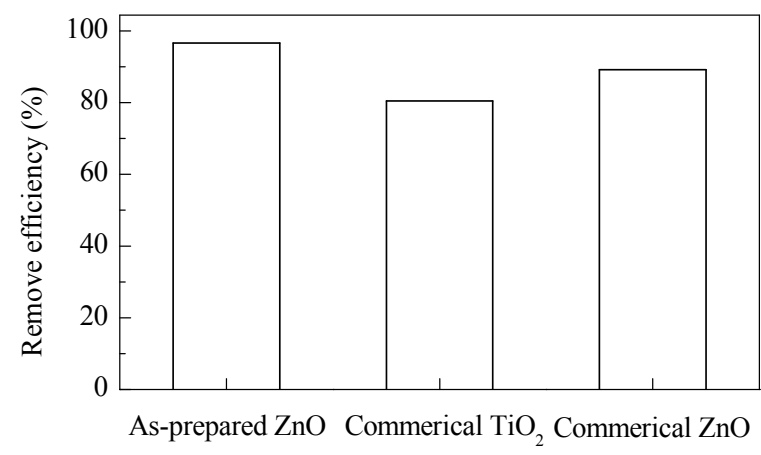

Fig. 12. Three successive CR photodegradation runs conducted using $\mathrm{ZnO}$.

\subsubsection{Stability and durability of the $\mathrm{ZnO}$ photocatalyst}

To evaluate the photostability of the catalyst, recycling experiments for the photodegradation of CR were performed and the results are shown in Fig. 12. We observed that $96.67 \%$ of the CR was degraded upon first $\mathrm{ZnO}$ use. However, after three recycles its photocatalytic activity decreased slightly and in the third run $91.14 \%$ of the CR degraded. Because of the facile dissolution of $\mathrm{ZnO}$ under extreme $\mathrm{pH}$ conditions as well as its photocorrosion, the photocatalytic tests were run under neutral pH conditions [39]. We also suggest that the smaller particle size of $\mathrm{ZnO}$ could have an important role in the inhibition of $\mathrm{ZnO}$ photocorrsion [40].

Furthermore, the XRD patterns of the $\mathrm{ZnO}$ nanostructures before and after 3 photoreaction cycles were also evaluated to confirm its stability. As shown in Fig. 13, the crystal structure of the produced $\mathrm{ZnO}$ did not change, which is indicative of its suitable stability.

\section{Conclusions}

The novel coordination compound $\mathrm{ZnC}_{12} \mathrm{H}_{10} \mathrm{~N}_{4} \mathrm{O}_{4}$ with a morphology that consists of hollow microblocks was synthesized by a simple chemical precipitation reaction. Calcination at $550{ }^{\circ} \mathrm{C}$ for $4 \mathrm{~h}$ provided a pure $\mathrm{ZnO}$ composition. Heat treatment of the precursor complex triggered the decomposition of the ligand, which in turn released volatile gases to produce

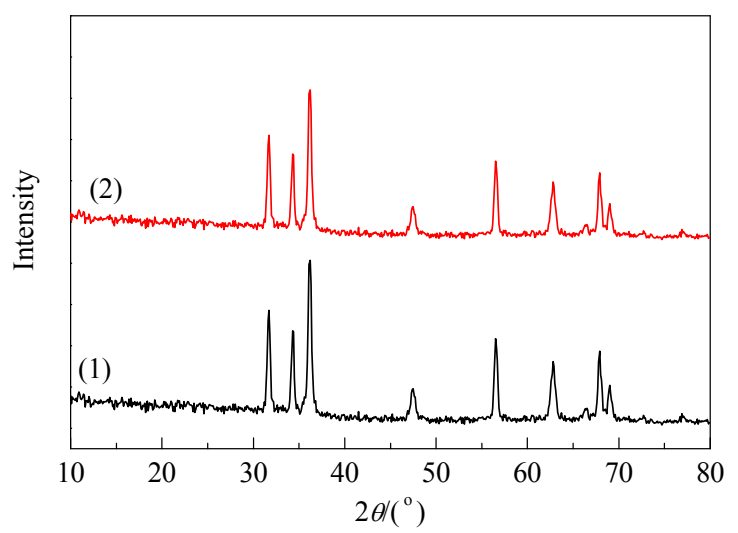

Fig. 13. XRD patterns of $\mathrm{ZnO}$ before (1) and after (2) 3 runs of the photocatalytic test. nanoparticles in the walls of the $\mathrm{ZnO}$ hollow microbricks. The mesoporous structure of the $\mathrm{ZnO}$ nanomaterials with a surface area of $8.13 \mathrm{~m}^{2} / \mathrm{g}$ and a pore size of $22.6 \mathrm{~nm}$ was determined by $\mathrm{N}_{2}$ adsorption-desorption analysis. The high photocatalytic activity of the produced $\mathrm{ZnO}$ nanostructure with special mesoporous morphology indicated that the product could efficiently catalyze the degradation of azo dyes.

\section{Acknowledgments}

This work was supported by Iran University of Science and Technology, the Research Council of Sharif University of Technology and Iran Nanotechnology Initiative Council.

\section{References}

[1] Rathnayake W G I U, Ismail H, Baharin A, Bandara I M C C D, Rajapakse S. J Appl Polym Sci, 2014, 131: 39601/1

[2] Akhavan O, Azimirad R, Safa S. Mater Chem Phys, 2011, 130: 598

[3] Akhavan 0, Mehrabian M, Mirabbaszadeh K, Azimirad R. J Phys D, 2009, 42: 225305/1

[4] Fang X, Wang X H, Zhao D X, Zhao H F, Fang F, Wei Z P, Li J H, Chu X Y, Wang F, Wang D D, Yan Y S. Physica E, 2014, 59: 93

[5] Nedic S, Chun Y T, Hong W K, Chu D P, Welland M. Appl Phys Lett, 2014, 104: 033101/1

[6] Chandiran A K, Abdi-Jalebi M, Nazeeruddin M K, Grätzel M. ACS Nano, 2014, 8: 2261

[7] Akhavan O.Carbon, 2011, 49: 11

[8] Kim M, Lee H J, Oh S, Kim Y, Jung H, Oh M K, Yoon Y J, Yoo T H, Yoon T S, Lee H H. Biosens Bioelectron, 2014, 56: 33

[9] Akhavan, O. ACS Nano, 2010, 4: 4174

[10] Zhang H, Sun J M, Dagle V L, Halevi B, Datye A K, Wang Y. ACS Catal, 2014, 4: 2379

[11] Alinezhad H, Tavakkoli S M, Biparva P. Chin J Catal (催化学报), 2014, 35: 560

[12] Lu J, Wang H H, Dong Y F, Wang F Q, Dong S J. Chin J Catal (催化学 报), 2014, 35: 1113

[13] Kumar R, Anandan S, Hembram K, Narasinga Rao T. ACS Appl Mater Interf, 2014, 6: 13138

[14] Wu D Z, Fan X M, Dai J, Liu H R, Liu H, Zhang F Z. Chin J Catal (催化 学报), 2012, 33: 802

[15] Wang X C, Yu J C, Ho C M, Hou Y D, Fu X Z. Langmuir, 2005, 21 : 2552

[16] Chong M N, Lei S M, Jin B, Saint C, Chow C W K. Sep Purif Technol, 2009, 67: 355

[17] Xu H L, Wang W Z, Zhu W.J Phys Chem B, 2006, 110: 13829

[18] Yu J G, Su Y R, Cheng B. Adv Funct Mater, 2007, 17: 1984

[19] Soares-Santos P C R, Sá Ferreira R A, Trindade T, Carlos L D, Nogueira H I S. J Alloys Compd, 2008, 451: 575

[20] Abu-Youssef M A M, Dey R, Gohar Y, Massoud A A, Öhrström L, Langer V. Inorg Chem, 2007, 46: 5893

[21] Toma M, Sanchez A, Garcia-Tasende M S, Casas J S, Castellano E E, Ellena J. Appl Organomet Chem, 2004, 18: 302

[22] Paraschiv C, Cucos A, Shova S, Madalan A M, Maxim C, Visinescu D, Cojocaru B, Parvulescu V I, Andruh M. Cryst Growth Des, 2014, DOI: $10.1021 / \operatorname{cg} 501604 c$

[23] Kimitsuka Y, Hosono E, Ueno S, Zhou H S, Fujihara S. Inorg Chem, 2013, 52: 14028

[24] Tadjarodi A, Izadi M, Imani M. Mater Lett, 2013, 92: 108

[25] Wang X Z, Liu W, Liu J R, Wang F L, Kong J, Qiu S, He C Z, Luan L Q. ACS Appl Mater Interf, 2012, 4: 817 


\section{Graphical Abstract}

Chin. J. Catal., 2015, 36: 742-749 doi: 10.1016/S1872-2067(14)60305-3

Photocatalytic activity of mesoporous microbricks of $\mathrm{ZnO}$ nanoparticles prepared by the thermal decomposition of bis(2-aminonicotinato) zinc (II)

Keyvan Bijanzad, Azadeh Tadjarodi, Omid Akhavan*

Iran University of Science and Technology, Iran; Sharif University of Technology, Iran; Sharif University of Technology, Iran
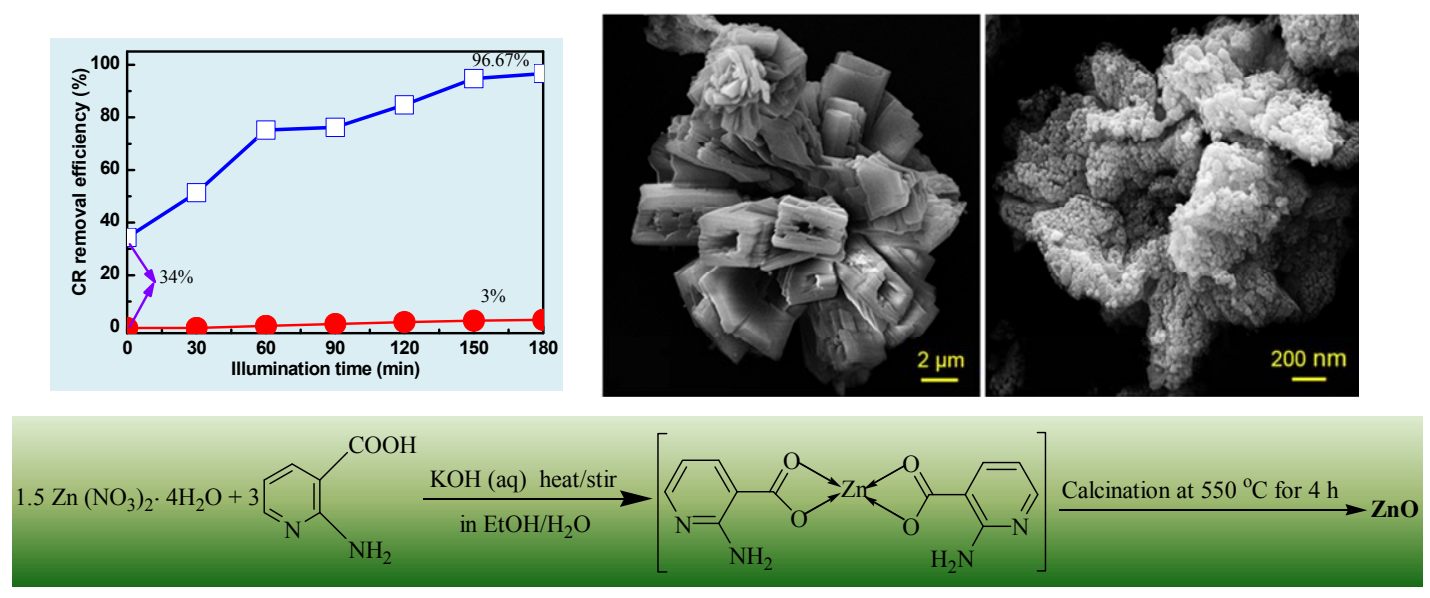

A novel zinc complex [Zn(anic)2] was used to produce a mesoporous $\mathrm{ZnO}$ nanoparticle structure by calcination at $550{ }^{\circ} \mathrm{C}$ for $4 \mathrm{~h}$. The $\mathrm{ZnO}$ nanoparticles showed efficient activity toward Congo red photodegradation.

[26] Jamal R K, Hameed M A, Adem K A. Mater Lett, 2014, 132: 31

[27] Jin C G, Yu T, Yang Y, Wu Z F, Zhuge L J, Wu X M, Feng Z C. Mater Chem Phys, 2013, 139: 506

[28] Sakkas V A, Islam Md A, Stalikas C, Albanis T A. J Hazard Mater, 2010, 175: 33

[29] Nakamoto K. Infrared and Raman Spectra of Inorganic and Coordination Compounds. 6th Ed. New Jersey: Wiley, 2009

[30] Zhang M Y, Liu Y, Li L, Gao H, Zhang X T. Catal Commun, 2015, 58: 122

[31] Zhou M J, Gao X H, Hu Y, Chen J F, Hu X. Appl Catal B, 2013, 138-139: 1

[32] Yu J G, Xiang Q J, Zhou M H. Appl Catal B, 2009, 90: 595

[33] Sivalingam G, Nagaveni K, Hegde M S, Madras G. Appl Catal B, 2003, 45: 23
[34] Yu J G, Yu J C, Leung M K P, Ho W, Cheng B, Zhao X J, Zhao J C. J Catal, 2003, 217: 69

[35] Cao L X, Spiess F J, Huang A M, Suib S L, Obee T N, Hay S O, Freihaut J D. J Phys Chem B, 1999, 103: 2912

[36] Xiao Q Si Z C, Zhang J, Xiao C, Tan X K. J Hazard Mater, 2008, 150: 62

[37] Huang Y, Ho W, Lee S, Zhang L Z, Li G S, Yu J C. Langmuir, 2008, 24: 3510

[38] Xiao K, Tian N, Guo Y X, Huang H W, Li X W, Zhang Y H. Inorg Chem Commun, 2015, 52: 5

[39] Comparelli R, Fanizza E, Curri M L, Cozzoli P D, Mascolo G, Agostiano A. Appl Catal B, 2005, 60: 1

[40] Zhang Y H, Chen Z, Liu S Q, Xu Y J. Appl Catal B, 2013, 140-141: 598 